\title{
Medical and Psychosocial Challenges Associated with Breast Cancer Survivorship
}

Raja Rahool ${ }^{1}$, Ghulam Haider ${ }^{1}$, Aisha Shahid ${ }^{1}$, Mehwish R. Shaikh ${ }^{1}$, Paras Memon ${ }^{1}$, Bhunisha Pawan ${ }^{1}$, Shumyla Beg ${ }^{1}$, Kiran Abbas ${ }^{2}$, Manahil Khalid ${ }^{3}$

1. Oncology, Jinnah Postgraduate Medical Centre, Karachi, PAK 2. Internal Medicine, Jinnah Postgraduate Medical Centre, Karachi, PAK 3. Internal Medicine, Bahria University Medical and Dental College, Karachi, PAK

Corresponding author: Kiran Abbas, kiranabbas2020@gmail.com

\section{Abstract \\ Objective}

To assess the association between common survivorship issues and characteristics of breast cancer survivors presenting at a tertiary care hospital in Karachi, Pakistan.

\section{Methodology}

This study was conducted in the medical oncology department of Jinnah Medical Postgraduate Center from March 27, 2019, to September 27, 2019. A number of 257 females of age group 18-90 years who had either completed their treatment or were undergoing treatment at the time were included using non-probability consecutive sampling techniques. Face-to-face interviews were personally conducted by the researcher, and data regarding the socio-demographics and common survivorship issues faced by breast cancer patients were obtained. The data acquired were entered and analyzed using SPSS version 23 (IBM Corp, Armonk, NY).

\section{Results}

The mean age of the breast cancer survivors were $42.58 \pm 10.07$ years. Of the main challenges, lack of energy received the highest mean score of $3.44 \pm 1.26$, followed by fatigue and financial issues. Overall the most common survivorship issue were financial issues ( $81.3 \%$ ), followed by fatigue ( $80.9 \%)$, cessation of the menstrual cycle (66.1\%), weak social support (59.1\%), and cosmetic disfigurement (51.8\%).

\section{Conclusion}

Breast cancer survivors have various psychological, medical, and social issues and may require unique attention during follow-up visits.

Review began 01/30/2021 Review ended 02/01/2021 Published 02/07/2021

\section{○ Copyright 2021}

Rahool et al. This is an open access article distributed under the terms of the Creative Commons Attribution License CC-BY 4.0., which permits unrestricted use, distribution, and reproduction in any medium, provided the original author and source are credited.
Categories: Psychology, General Surgery, Oncology

Keywords: breast neoplasms, financial stress, survivorship, social support, mastectomy, psycho-oncology, psychophysiology, cancer survivors

\section{Introduction}

One of the most prevalent malignancies among females is breast cancer. Globally, in 2018 the incidence of breast cancer was estimated as $25 \%$ of all cancers [1]. Within the last 10 years, the incidence of breast cancer has increased by $6 \%$, mortality rates have decreased significantly and at present, $80 \%$ of early breast cancer patients survive for more than 10 years [2]. In 2010, there were an estimated 500,000 breast cancer survivors and by 2040 it is expected to cross two million survivors [3]. The five-year survival rate of breast cancer differs from country to country. In India, the survival rate was estimated as $60 \%$, in Malaysia as $68 \%$, in South Africa as 53\%, in Mongolia as 57\% and in Oceania and North America up to 89\% [4].

The rate of survival of breast cancer patients has been significantly enhanced with improvements and availability of therapy. However, literature has shown that women with breast cancer who have undergone chemotherapy, radiotherapy, biotherapy or hormonal therapy, surgery, and reconstructive surgery may face numerous psychosocial and physical issues and as well as a compromised overall quality of life [5].

After adjuvant therapy, most of the women encounter weight gain, musculoskeletal pain, hot flashes, nausea, and itching around the genital area. [6] The sexual desire of breast cancer survivors is also highly affected after therapy and may experience fatigue, loss of nipple sensitivity, vaginal dryness, and scarring [6]. Psychological problems such as sadness, anxiety, and depression owing to therapy, recurrence, and bodily disfigurement stay constant after diagnosis and cure. Besides, the lack of support and communication from their partners, family, and friends reduced their social integration [5, 7]. Survivors of breast cancer are also at high risk of secondary health issues such as cardiovascular disease, cessation of the menstrual cycle, and poor bone health (osteoporosis) [8]. Other common issues are low physical activity and poor dietary 
habits, which are associated with recurrence of breast cancer [9]. The majority of breast cancer survivors are unable to rejoin their respective occupations due to their inability to work. This is associated with a high financial burden because the resources to pay consultation fees, medical bills, and medications become limited, therefore they show poorer medication adherence [10].

As the survival rate of breast cancer is increasing, the long-term social, physical, and psychological issues are also becoming very common among them. There is no data available in Pakistan addressing the most common issues which have a significant impact on the quality of their life. So, the current research aimed to assess the common survivorship issues faced by breast cancer patients. This research would be helpful for health professionals to better understand the needs of recovered patients, identify issues and develop strategies to resolve them so that women can have a good quality of life.

\section{Materials And Methods}

It was a cross-sectional study performed at the department of the medical oncology department of Jinnah Medical Postgraduate Center from March 2019 to September 2019. The sample size of 257 was estimated using the OpenEpi online sample size calculator by taking proportion as 0.28 [5] of vaginal dryness among breast cancer survivors, absolute precision as 5.5\%, and 95\% confidence level. All the females of age 18-90 years who had completed their treatment and who were on treatment were included using the nonprobability consecutive sampling technique. Women with psychiatric problems, having pregnancy, a history of recurrent disease, and who didn't give consent were excluded from the study.

Approval from the hospital's ethical review committee was sought before data collection and informed written or verbal consent was taken from all the eligible females. Face-to-face interviews were personally conducted by the researcher and information was noted in the questionnaire while maintaining the confidentiality and privacy of data.

The questionnaire was divided into two parts, the first part included the questions regarding the sociodemographic properties of the participants and the second part included the questions regarding the common survivorship issues faced by breast cancer patients. The issues were evaluated using "Breast cancer prevention trial symptoms scale (BCPT)" and "Cancer rehabilitation evaluation system (CARES)" [11-13]. The BCPT scale assessed the symptoms such as fatigue, nausea, hot flashes, cognitive problems, musculoskeletal pain, weight problems, vaginal problem, bladder problem, arm problems, and sexual interest whereas the CARE scale was used to evaluate the psychological issues. Furthermore, medical issues such as any cardiovascular problem, hormonal blockade, and osteoporosis within the last one month were assessed using the medical history of the patients. The questions regarding the weak family support, financial strain and cosmetic issues were also asked. The females reported the severity of each problem experienced by them in the last one month on a five-point Likert scale of 0 (not all) to 4 (extremely). The cutoff scores for bladder and vaginal problems, as well as other medical and social issues, were taken as $\geqslant 4$; for hot flashes, weight and arm problems, fatigue and sexual interest as $\geqslant 6$; for musculoskeletal and cognitive problems as $\geqslant 8$, and for psychological problems as $\geqslant 12$. The final questionnaire was translated into Urdu and pre-testing of the final questionnaire was done in 10 survivors of breast cancer and Cronbach's alpha value was calculated as 0.72 .

All the data was entered and analyzed using SPSS version 23 (IBM Corp., Armonk, NY). Quantitative variables were described as mean and SD, while qualitative variables were displayed as frequency and proportion. The common survivorship issues were stratified with respect to age, residence, socioeconomic status, education, and marital status. The post-stratification chi-square test was applied. The p-value $<0.05$ was considered as statistically significant.

\section{Results}

The mean age of the 257 breast cancer survivors was $42.90 \pm 10.39$ years. The mean BMI was reported as $26.90 \pm 5.96 \mathrm{~kg} / \mathrm{m} 2$. The majority of the patients were living in urban areas $(73.9 \%)$ whereas 67 were from rural areas $(26.1 \%)$. Out of 257 , most of them were illiterate $(n=90 ; 35 \%)$ followed by primary $(n=81 ; 31.5 \%)$, secondary $(n=49 ; 19.1 \%)$, intermediate $(n=22,8.6 \%)$ and graduate $(n=15,5.8 \%)$. Also, $185(72 \%)$ had low socioeconomic status (monthly income $<15,000$ rupees), 67 (26.1\%) had middle socioeconomic status (monthly income 15,000-30,000 rupees) and only five (1.9\%) had high socioeconomic status (monthly income $>30,000$ rupees). The majority of the females were married $(n=210,81.7 \%)$ whereas $47(18.3 \%)$ were unmarried. Individual responses and mean scores of breast cancer survivorship issues by study participants are displayed in Table 1.

\begin{tabular}{|c|c|c|c|c|c|c|}
\hline Survivorship Issues & Not at all (0) & A little (1) & Fair (2) & Much (3) & Very much (4) & Mean \pm SD \\
\hline \multicolumn{7}{|l|}{ Hot flashes } \\
\hline a. Hot flashes & $74(28.8 \%)$ & $7(2.7 \%)$ & $37(14.4 \%)$ & $38(14.8 \%)$ & $101(39.3 \%)$ & $2.33 \pm 1.67$ \\
\hline b. Night sweats & $116(45.1 \%)$ & $4(1.6 \%)$ & $18(1.0 \%)$ & $3 /(14.4 \%)$ & $82(31.9 \%)$ & $1.86 \pm 1.8$ \\
\hline
\end{tabular}




\section{Cureus}

\begin{tabular}{|c|c|c|c|c|c|c|}
\hline Bladder control & & & & & & \\
\hline Loss of bladder control & $175(68.1 \%)$ & $4(1.6 \%)$ & $11(4.3 \%)$ & $14(5.4 \%)$ & $53(20.6 \%)$ & $1.09 \pm 1.67$ \\
\hline Vaginal problems & & & & & & \\
\hline Vaginal dryness & $108(42 \%)$ & $9(3.5 \%)$ & $52(20.2 \%)$ & $16(6.2 \%)$ & $72(28 \%)$ & $1.75 \pm 1.69$ \\
\hline Musculoskeletal problems & & & & & & \\
\hline a. General aches and pain & $71(27.6 \%)$ & $2(0.8 \%)$ & $8(3.1 \%)$ & $15(5.8 \%)$ & $161(62.6 \%)$ & $2.75 \pm 1.77$ \\
\hline b. Joint pain & $188(73.2 \%)$ & $5(1.9 \%)$ & $0(0.0 \%)$ & $4(1.6 \%)$ & $60(23.3 \%)$ & $1 \pm 1.7$ \\
\hline c. Muscle stiffness & 194(75.5\%) & $5(1.9 \%)$ & $0(0.0 \%)$ & $4(1.6 \%)$ & $54(21 \%)$ & $0.91 \pm 1.65$ \\
\hline Weight problems & & & & & & \\
\hline a. Unhappy with appearance of owns body & $139(54.1 \%)$ & $10(3.9 \%)$ & $22(8.6 \%)$ & $20(7.8 \%)$ & $66(25.7 \%)$ & $1.47 \pm 1.74$ \\
\hline b. Weight gain & $169(65.8 \%)$ & $2(0.8 \%)$ & $6(2.3 \%)$ & $15(5.8 \%)$ & $65(25.3 \%)$ & $1.24 \pm 1.77$ \\
\hline Cognitive problems & & & & & & \\
\hline a. Forgetfulness & $166(64.6 \%)$ & $8(3.1 \%)$ & $7(2.7 \%)$ & $18(7 \%)$ & $58(22.6 \%)$ & $1.2 \pm 1.72$ \\
\hline b. Difficulty concentrating & $153(59.5 \%)$ & $21(8.2 \%)$ & $10(3.9 \%)$ & $6(2.3 \%)$ & $67(26.1 \%)$ & $1.27 \pm 1.74$ \\
\hline c. Easily distracted & $185(72 \%)$ & $5(1.9 \%)$ & $11(4.3 \%)$ & $15(5.8 \%)$ & $41(16 \%)$ & $0.92 \pm 1.56$ \\
\hline
\end{tabular}

Arm problems

b. Arm swelling

$203(79 \%)$

$0(2.3 \%)$

$7(2.7 \%)$

$11(4.3 \%)$

$47(18.3 \%)$

$0.94 \pm 1.6$

$10(3.9 \%) \quad 9(3.5 \%) \quad 29(11.3 \%)$

$0.66 \pm 1.37$

Fatigue
a. Tiredness
b. Lack of energy

$26(10.1 \%)$

0(0.0\%) $\quad 9(3.5 \%)$

$43(16.7 \%) \quad 179(69.6 \%)$

$3.36 \pm 1.23$

$33(12.8 \%)$

$0(0.0 \%)$

$3(1.2 \%)$

$24(9.3 \%) \quad 197(76.7 \%)$

$3.37 \pm 1.34$

\section{Sexual interest}

a. Lack of interest in sex

b. Low sexual enjoyment

$73(28.4 \%)$

$28(10.9 \%) \quad 33(12.8)$

$18(7 \%)$

$105(40.9 \%)$

$2.21 \pm 1.71$

$96(37.4 \%)$

$28(10.9 \%) \quad 36(14 \%)$

$44(17.1 \%) \quad 53(20.6 \%)$

$1.73 \pm 1.59$

\section{Psychological problems}

a. Felt worried and anxious about future

$85(33.1 \%)$

$5(1.9 \%) \quad 8(3.1 \%)$

$31(12.1 \%) \quad 128(49.8 \%)$

$2.44 \pm 1.81$

b. Felt despair about health

$71(27.6 \%)$

$0(0.0 \%)$

$10(3.9 \%)$

$27(10.5 \%) \quad 149(58 \%)$

$2.71 \pm 1.74$

c. Felt sad depressed and lost interest

119(46.3\%)

$17(6.6 \%)$

$29(11.3 \%) \quad 31(12.1 \%) \quad 61(23.7 \%)$

$1.6 \pm 1.69$

d. Felt angry and irritable

$123(47.9 \%)$

$10(3.9 \%)$

$27(10.5 \%) \quad 29(11.3 \%) \quad 68(26.5 \%)$

$1.65 \pm 1.74$ 


\section{Cureus}

\begin{tabular}{|c|c|c|c|c|c|c|}
\hline \multicolumn{7}{|l|}{ Other medical issues } \\
\hline a. Cardiovascular issue & $184(71.6 \%)$ & $31(12.1 \%)$ & $18(7 \%)$ & $4(1.6 \%)$ & $20(7.8 \%)$ & $0.62 \pm 1.19$ \\
\hline b. Cessation of menstrual cycle & $56(21.8 \%)$ & $18(7.0 \%)$ & $8(3.1 \%)$ & $5(1.9 \%)$ & $170(66.1 \%)$ & $2.84 \pm 1.71$ \\
\hline c. Osteoporosis & $136(52.9 \%)$ & $35(13.6 \%)$ & $14(2 \%)$ & $2(0.8 \%)$ & $70(27.2 \%)$ & $1.36 \pm 1.72$ \\
\hline \multicolumn{7}{|l|}{ Other social issues } \\
\hline a. Financial issue & $36(14 \%)$ & $7(2.7 \%)$ & $3(1.2 \%)$ & $2(0.8 \%)$ & 209(81.3\%) & $3.33 \pm 1.45$ \\
\hline b. Weak family support & $68(26.5 \%)$ & $5(1.9 \%)$ & $12(4.7 \%)$ & $24(9.3 \%)$ & $148(57.6 \%)$ & $2.7 \pm 1.73$ \\
\hline c. Cosmetic issue & $110(42.8 \%)$ & $3(1.2 \%)$ & $6(2.3 \%)$ & $5(1.9 \%)$ & $133(51.8 \%)$ & $2.19 \pm 1.95$ \\
\hline
\end{tabular}

TABLE 1: Mean scores and distribution of breast cancer survivorship issues in study participants.

The lack of energy recorded the highest mean score at $3.44 \pm 1.26$, followed by fatigue and financial issues. Overall the most common survivorship issue was financial issues $(n=209 ; 81.3 \%)$, followed by fatigue $(n=08$; $80.9 \%)$, cessation of menstrual cycle ( $\mathrm{n}=170 ; 66.1 \%)$, weak social support $(\mathrm{n}=152 ; 59.1 \%)$, and cosmetic disfigurement ( $\mathrm{n}=133 ; 51.8 \%)$ as displayed in Figure 1 .

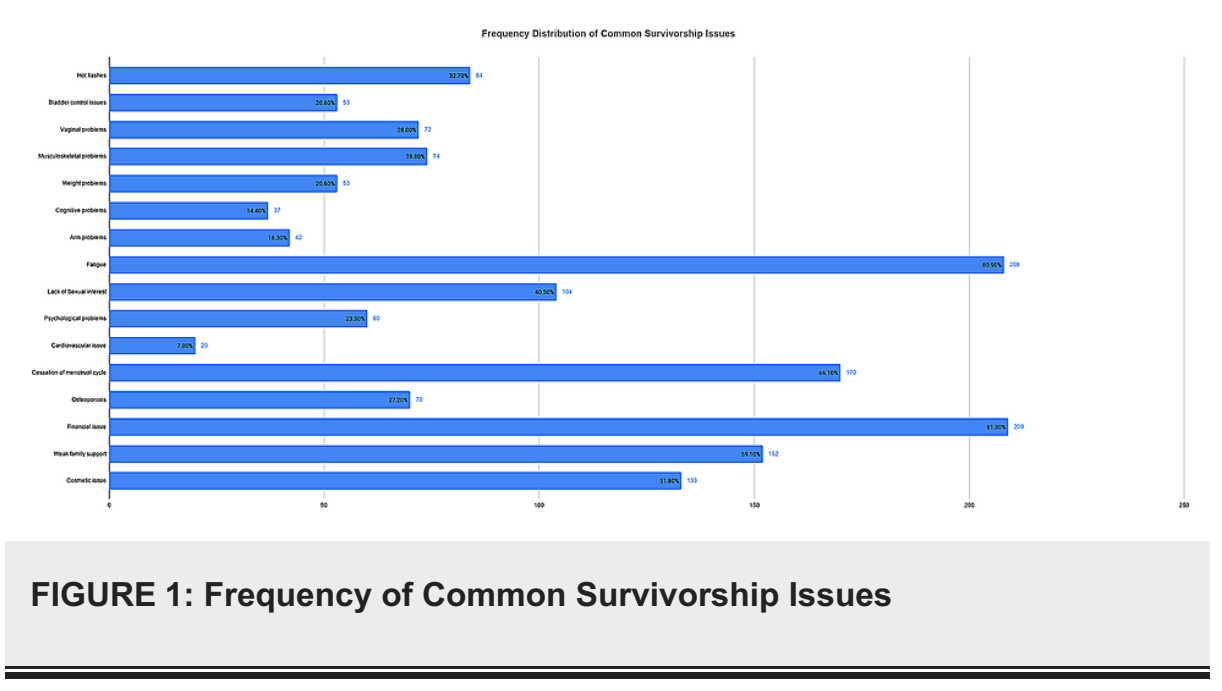

Stratification with respect to age, residence, educational status, socioeconomic status, and marital status was done to see the effect on survivorship issues. The issue of hot flashes showed a statistically significant relationship with age, educational status, and marital status ( $\mathrm{p}<0.05)$. Bladder problems showed a statistically significant relationship with age, residence, and marital status ( $\mathrm{p}<0.05)$. The vaginal, musculoskeletal, and weight problems, as well as cessation of menstrual cycle showed a statistically significant relationship with age, residence, and educational status ( $\mathrm{p}<0.05)$. Cognitive and arm problems showed a statistically significant relationship with age and residence $(\mathrm{p}<0.05)$. Fatigue showed a statistically significant relationship with age, educational status, socio-economic status, and marital status $(\mathrm{p}<0.05)$. Lack of sexual interest was statistically significant with respect to age and marital status $(\mathrm{p}<0.05)$. Psychological issues showed a statistically significant relationship with educational status ( $\mathrm{p}<0.05)$. Osteoporosis showed a statistically significant relationship with residence and educational status $(\mathrm{p}<0.05)$. Financial stress showed a statistically significant relationship with educational status, socio-economic status, and marital status $(\mathrm{p}<0.05)$. Weak social support showed a statistically significant relationship with educational status and socio-economic status $(\mathrm{p}<0.05)$. Cosmetic disfigurement showed a statistically significant relationship with educational status $(\mathrm{p}<0.05)$. More stratifications are mentioned in Table 2. 


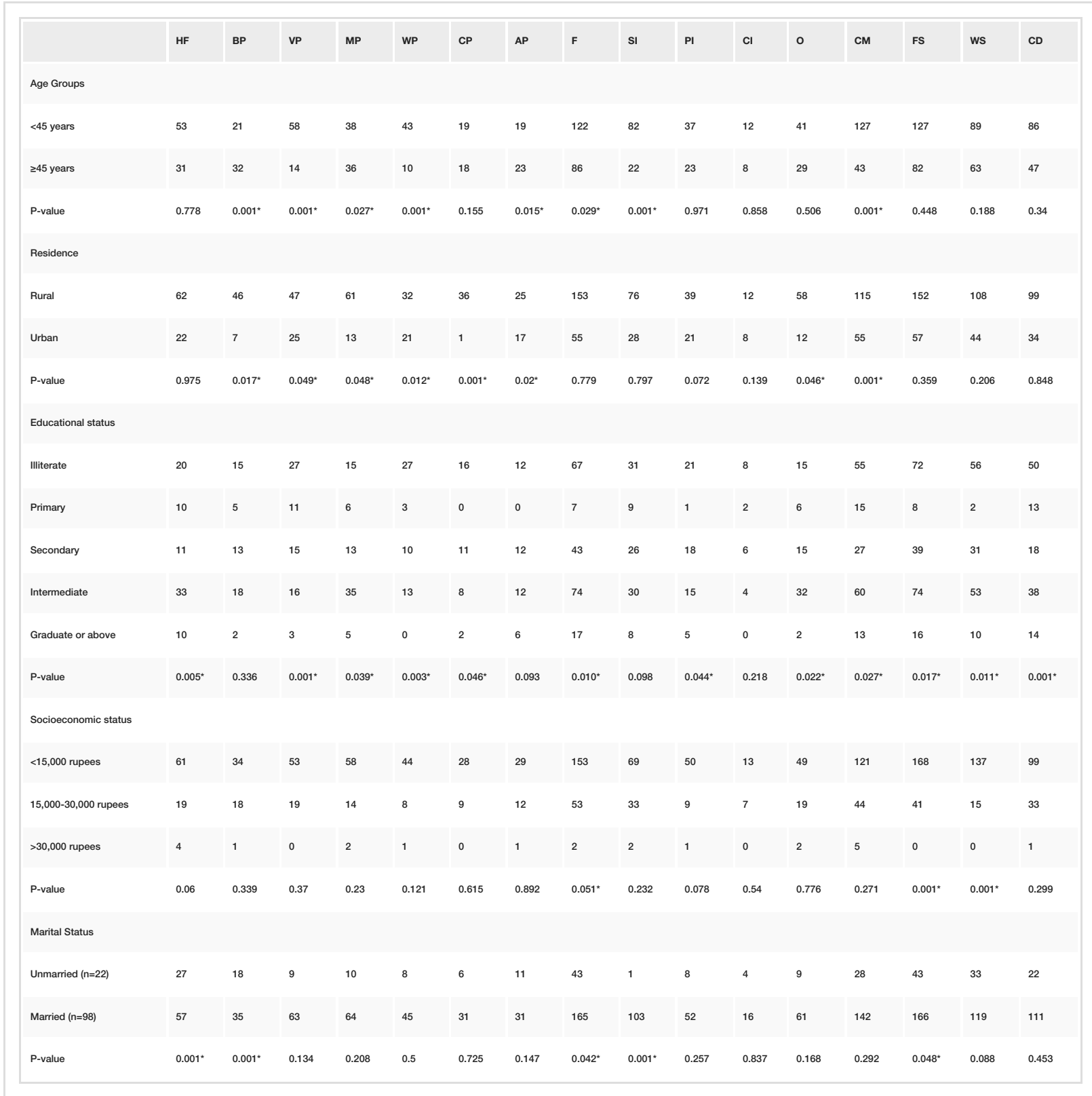

\section{TABLE 2: Stratification of Common Survivorship Issues with Patient Characteristics}

$\mathrm{HF}=$ Hot flash, $\mathrm{BP}=\mathrm{Bladder}$ problem, $\mathrm{VP}=$ Vaginal problem, $\mathrm{MP}=$ Musculoskeletal problem, WP=Weight problem, $\mathrm{CP}=\mathrm{Cognitive} \mathrm{problem,} \mathrm{AP}=\mathrm{Arm}$ problem, $\mathrm{F}=$ Fatigue, $\mathrm{SI}=$ Sexual interest, $\mathrm{Pl}=\mathrm{Psychological}$ issues, $\mathrm{Cl}=$ Cardiac issues, $\mathrm{O}=$ Osteoporosis, $\mathrm{CM}=\mathrm{Cessation}$ of menstrual cycle,

$\mathrm{FS}=$ Financial stress, WS=Weak social support, $\mathrm{CD}=$ Cosmetic disfigurement, ${ }^{*}=$ significant $\mathrm{p}<0.05$

\section{Discussion}

Most survivors after and during breast cancer treatment delight in their longer lifespan, but there is currently little knowledge accessible from their view about the most prevalent and important problems they face. Hence, the current study explored the challenges faced by breast cancer survivors and its association with different factors.

In Asia, developing countries have overcrowded and scarce resources in public hospitals so patients mostly prefer private hospitals for better management. Therefore, the treatment and follow-up is very costly. The majority of the breast cancer survivors struggle with financial stress [14]. In a study by Pietrangelo, about $36 \%$ of the women reported being unable to continue their job and lose their careers after surviving breast cancer [15]. In the current study financial issues were the most frequent challenge associated with survivorship among breast cancer patients. The financial constraints were associated with the educational status, socioeconomic background, and marital status of the survivors $(\mathrm{p}<0.05)$. In the results of the longitudinal research on breast cancer survivors, it was shown that most of them encountered economic hardships, where out of pocket expenses were $40 \%$ among participants with $<\$ 500$ expenditure [16]. In 
another research by Fenn in the US, 18\% of breast cancer survivors had financial issues and showed a statistically significant association with family income $(\mathrm{p}<0.05)$ [17]. A study conducted in Pakistan showed that $35 \%$ of the breast cancer survivors belonged to low socioeconomic groups and had five-year diseasefree and overall survival of $31 \%$ and $45 \%$ respectively $(\mathrm{p}<0.05)$ [18].

Another challenge faced by survivors was chronic fatigue ranging from $4 \%$ to $91 \%$, mainly influencing the quality of life. Fatigue was noted in $50 \%$ of the patients after the first five years of therapy [19]. This was in accordance with our study. In a meta-analysis, the prevalence of severe fatigue was reported as $7-52 \%$ in breast cancer survivors with pooled prevalence as $27 \%$ [20].

The hormonal therapies have shown a significant reduction in the rate of mortality and recurrence rate by $30 \%$ and $31 \%$ respectively [21]. However, the most common side effects of these therapies are reported as vaginal dryness and hot flashes. In addition, it is also associated with the cessation of the menstrual cycle [22]. In the current research, vaginal dryness, and cessation of menstrual cycle were reported in association with age, residence, and education level $(\mathrm{p}<0.05)$. In previous research, almost half of the breast cancer survivors had reported at least one hot flash in the past 1 day (45\%) or past seven days (52\%) and the majority of them had mild to asymptomatic severity [23]. In another similar research by Sapkota et al. conducted in Nepal, the frequency of hot flashes was reported as $45 \%$ and the mean rank for hot flashes was higher in females who had completed their treatment within 1 year. In addition, the percentage of vaginal problems was reported as $28 \%$ among them [5].

In Asia, heavily patriarchal culture is present, the final verdict is made by the family's head. Their assistance and choice to go for therapy is therefore very important in breast cancer management. In such situations, if the family is not supportive, patients suffer from psychosocial distress and mental complications [7]. Therefore, strong emotional \& social support from family and friends play a vital role in the life of breast cancer survivors. In Pakistan, a study by Banning et al. showed that initially, family support is present however later on it becomes inconsiderate for the patient's family. The patients showed more concerns regarding their children, husband, and family, rather than their own selves [24]. In the current research, weak social support was observed in $59.1 \%$ of the females and it was more in low socioeconomic status and illiterate females $(\mathrm{p}<0.05)$. In the previous study, social problems were one of the significant issues among breast cancer survivors and had a high mean rank score of 23.9 among women of age $<45$ years [5]. This is similar to the present study, young women ( $<45$ years) had a high frequency of weak social support.

Breast cancer treatment involves mastectomy that has side effects such as scarring, weight gain, body dysfunction, and cosmetic disfigurement that have universal female sexuality and womanhood connotations. The change in body image often leads to dissatisfaction and bodily shame and low self-esteem [9]. Bodily disfigurement and sexual dysfunction have a major influence on psychological as physiological aspects of breast cancer survivors. It also affected the sex life of their partners [9]. As young breast cancer patients are more sexually active, lack of sexuality plays an important role [25]. The intrapsychic capacity of females changes due to a sexual dysfunction that involves negative body image, fear of fertility loss, loss of femininity, absence of sexual interest, and shifts to a sense of sexual self [25]. In the present study, the majority of the breast cancer survivors lack their interest in sex due to sexual dysfunction and it was prevalent among age groups less than 45 years $(\mathrm{p}<0.05)$. The married females had more sexual dysfunctionality as compared to unmarried and showed statistically significant differences $(\mathrm{p}<0.05)$. Furthermore, the frequency of cosmetic disfigurement was $51.8 \%$. In a previous study, the breast cancer survivors reported poor sexual dysfunction and sexual satisfaction as $45 \%$ and $44 \%$ respectively. About $81 \%$ of them showed less sexual sense/desire and $43 \%$ of them presented with dyspareunia. Furthermore, $42 \%$ of them had anxiety and $44 \%$ had depression which was significantly associated with body image $(p<0.05)[25]$.

\section{Conclusions}

The present study offers insight into the challenges faced by survivors of breast cancer. It is important to keep a strict follow-up after completion of treatment. The clinicians should assess whether the survivors need counseling from a professional psychologist or any lifestyle modifications. Future studies should focus on the impact of small support group meetings for overcoming the survivor's medical and psychological issues.

\section{Additional Information \\ Disclosures}

Human subjects: Consent was obtained or waived by all participants in this study. College of Physicians and Surgeons Pakistan issued approval Onc-7833. The approval from the hospital's ethical review committee was sought before data collection. Informed written or verbal consent was taken from all the eligible females. The face to face interviews were personally conducted by the researcher and information was noted in the questionnaire while maintaining the confidentiality and privacy of data. . Animal subjects: All authors have confirmed that this study did not involve animal subjects or tissue. Conflicts of interest: In compliance with the ICMJE uniform disclosure form, all authors declare the following: Payment/services info: All authors have declared that no financial support was received from any organization for the 
submitted work. Financial relationships: All authors have declared that they have no financial relationships at present or within the previous three years with any organizations that might have an interest in the submitted work. Other relationships: All authors have declared that there are no other relationships or activities that could appear to have influenced the submitted work.

\section{References}

1. WCRF: Worldwide cancer data, Global cancer statistics for the most common cancers . (2018). Accessed: January 10, 2021: https://www.wcrf.org/dietandcancer/cancer-trends/worldwide-cancer-data..

2. Rowntree C, Duke V, Panayiotidis P, Kotsi P, Palmisano GL, Hoffbrand AV, Foroni L: Deletion analysis of chromosome 13q14. 3 and characterisation of an alternative splice form of LEU1 in B cell chronic lymphocytic leukemia. Leukemia. 2002, 16:1267. 10.1038/sj.leu.2402551

3. Maddams J, Utley M, Moller H.: Projections of cancer prevalence in the United Kingdom, 2010-2040 . Br J Cancer. 2012, 107:1195-202. 10.1038/bjc.2012.366

4. Begum N: Breast cancer in Pakistan: a looming epidemic . J Coll Physicians Surg Pak. 2018, 28:87-8.

5. Sapkota A, Shrestha S, Sedhain A, Koirala S, Kafle P: Problems of breast cancer survivors living in an urban area of Nepal. Asia Pac J Oncol Nurs. 2016, 3:289-96. 10.4103/2347-5625.189818

6. Chang YC, Hu WY, Chang YM, Chiu SC: Changes in sexual life experienced by women in Taiwan after receiving treatment for breast cancer. Int J Qual Stud Health Well-being. 2019, 14:1. 10.1080/17482631.2019.1654343

7. Weis J: Psychosocial care for cancer patients. Breast Care (Basel). 2015, 10:84-6. 10.1159/000381969

8. National Research Council: From Cancer Patient to Cancer Survivor: Lost in Transition . National Academies Press, Washington, D.C; 2006. 10.17226/11468

9. Cho J, Jung SY, Lee JE, et al.: A review of breast cancer survivorship issues from survivors' perspectives . J Breast Cancer. 2014, 17:189-99. 10.4048/jbc.2014.17.3.189

10. Altice CK, Banegas MP, Tucker-Seeley RD, Yabroff KR: Financial hardships experienced by cancer survivors: a systematic review. J Natl Cancer Inst. 2017, 109:2. 10.1093/jnci/djw205

11. Stanton AL, Bernaards CA, Ganz PA: The BCPT symptom scales: a measure of physical symptoms for women diagnosed with or at risk for breast cancer. J Natl Cancer Inst. 2005, 97:448-56. 10.1093/jnci/dji069

12. Alfano CM, McGregor BA, Kuniyuki A, et al.: Psychometric properties of a tool for measuring hormonerelated symptoms in breast cancer survivors. Psychooncology. 2006, 15:985-1000. 10.1002/pon.1033

13. Kumar SP, D'Souza M, Sisodia V, Kumar KV: Cancer rehabilitation evaluation system questionnaire: a disease-specific and treatment-specific measure of rehabilitation needs and self-reported quality of life. Indian J Palliat Care. 2014, 20:243-4. 10.4103/0973-1075.138403

14. Bajpai V: The challenges confronting public hospitals in India, their origins, and possible solutions . Advances in Public Health. 2014, 27:243-4. 10.1155/2014/898502

15. Pietrangelo A. Breast cancer survivors have a bigger problem after treatment: the cost . (2018). Accessed: February 6, 2021: https://www.healthline.com/health-news/breast-cancer-survivors-have-problem-aftertreatment-the-cost\#1..

16. Jagsi R, Pottow JA, Griffith KA, et al.: Long-term financial burden of breast cancer: experiences of a diverse cohort of survivors identified through population-based registries. J Clin Oncol. 2014, 32:1269-76. 10.1200/JCO.2013.53.0956

17. Fenn K. Is the financial burden of cancer impacting survivors' quality of life? . (2014). Accessed: February 6 , 2021: https://elischolar.library.yale.edu/cgi/viewcontent.cgi?article=1875\&context=ymtdl.

18. Aziz Z, Sana S, Akram M, Saeed A: Socioeconomic status and breast cancer survival in Pakistani women . J Pak Med Assoc. 2004, 54:448-53.

19. Tabrizi FM, Alizadeh S: Cancer related fatigue in breast cancer survivors: in correlation to demographic factors. Maedica (Bucur). 2017, 12:106-11.

20. Abrahams HJ, Gielissen MF, Schmits IC, Verhagen CA, Rovers MM, Knoop H: Risk factors, prevalence, and course of severe fatigue after breast cancer treatment: a meta-analysis involving 12327 breast cancer survivors. Ann Oncol. 2016, 27:965-74. 10.1093/annonc/mdw099

21. Tremont A, Lu J, Cole JT: Endocrine therapy for early breast cancer: updated review . Ochsner J. 2017, 17:405-11.

22. Bodai BI, Tuso P: Breast cancer survivorship: a comprehensive review of long-term medical issues and lifestyle recommendations. Perm J. 2015, 19:48-79. 10.7812/TPP/14-241

23. Chang HY, Jotwani AC, Lai YH, Jensen MP, Syrjala KL, Fann JR, Gralow J: Hot flashes in breast cancer survivors: frequency, severity and impact. Breast. 2016, 27:116-21. 10.1016/j.breast.2016.02.013

24. Banning M, Hafeez H, Faisal S, Hassan M, Zafar A: The impact of culture and sociological and psychological issues on Muslim patients with breast cancer in Pakistan. Cancer Nurs. 2009, 32:317-24. 10.1097/NCC.0b013e31819b240f

25. Leila M, Nada C, Kais C, Jawaher M: Sexuality after breast cancer: cultural specificities of Tunisian population. Pan Afr Med J. 2016, 25:17-24. 10.11604/pamj.2016.25.17.10399 